\title{
ARTICLE
}

Received 3 Dec 2014 | Accepted 17 Jun 2016 | Published 28 Jul 2016

DOI: $10.1038 /$ ncomms12267

OPEN

\section{Synthesis of sodium polyhydrides at high pressures}

\author{
Viktor V. Struzhkin ${ }^{1}$, Duck Young Kim¹,2, Elissaios Stavrou1,3, Takaki Muramatsu' ${ }^{1}$, Ho-kwang Mao 1,2, \\ Chris J. Pickard ${ }^{4,5}$, Richard J. Needs ${ }^{6}$, Vitali B. Prakapenka ${ }^{7}$ \& Alexander F. Goncharov ${ }^{1,8}$
}

The only known compound of sodium and hydrogen is archetypal ionic $\mathrm{NaH}$. Application of high pressure is known to promote states with higher atomic coordination, but extensive searches for polyhydrides with unusual stoichiometry have had only limited success in spite of several theoretical predictions. Here we report the first observation of the formation of polyhydrides of $\mathrm{Na}\left(\mathrm{NaH}_{3}\right.$ and $\left.\mathrm{NaH}_{7}\right)$ above $40 \mathrm{GPa}$ and 2,000 K. We combine synchrotron $\mathrm{X}$-ray diffraction and Raman spectroscopy in a laser-heated diamond anvil cell and theoretical random structure searching, which both agree on the stable structures and compositions. Our results support the formation of multicenter bonding in a material with unusual stoichiometry. These results are applicable to the design of new energetic solids and high-temperature superconductors based on hydrogen-rich materials.

\footnotetext{
${ }^{1}$ Geophysical Laboratory, Carnegie Institution of Washington, 5251 Broad Branch Road NW, Washington, District of Columbia 20015, USA. ${ }^{2}$ Center for High Pressure Science and Technology Advanced Research, Shanghai 201203, China. ${ }^{3}$ Lawrence Livermore National Laboratory, Material Sciences Division, 7000 East Avenue, L-350, Livermore, CA 94550-9698, USA. ${ }^{4}$ Department of Physics and Astronomy, University College London, Gower Street, London WC1E 6BT, UK. ${ }^{5}$ Department of Materials Science \& Metallurgy, University of Cambridge, 27 Charles Babbage Road, Cambridge CB3 OFS, UK. ${ }^{6}$ Theory of Condensed Matter Group, Cavendish Laboratory, J J Thomson Avenue, Cambridge CB3 OHE, UK. ${ }^{7}$ Center for Advanced Radiation Sources, The University of Chicago, Chicago, Illinois 60637, USA. ${ }^{8}$ Key Laboratory of Materials Physics, Institute of Solid State Physics, Chinese Academy of Sciences, Hefei, Anhui 230031, China. Correspondence and requests for materials should be addressed to V.V.S. (email: vstruzhkin@carnegiescience.edu).
} 
$\mathrm{D}$ ense hydrogen is of central interest in many disciplines, especially in high-pressure science. It is expected to possess unusual properties such as high energy density ${ }^{1}$, high-temperature superconductivity and superfluidity ${ }^{2}$. Unusual high-pressure properties may be sustained at ambient conditions, if a predicted metastable metallic phase of hydrogen ${ }^{3}$ could exist at ambient pressure. This phase would have unusual anisotropic structure, consisting of weakly interacting chains of hydrogen atoms with interatomic distances $\sim 1.06 \AA$ (ref. 3). Looking for another route to force hydrogen into a metallic state, Ashcroft ${ }^{4}$ proposed that such conducting states could be realized in hydrogen-rich alloys, where hydrogen is in a 'pre-compressed' or otherwise altered electronic states induced by the host material ('dopant') in such a way, that the electronic bands of hydrogen and the host element(s) overlap at the Fermi level. For example, polyhydrides $\mathrm{LiH}_{2}$ and $\mathrm{LiH}_{6}$ (refs 5,6) were predicted to have stable semi-metallic $\left(\mathrm{LiH}_{2}\right)$ and metallic $\left(\mathrm{LiH}_{6}\right)$ phases above $100 \mathrm{GPa}$, which is nearly four times lower than the calculated metallization pressure of pure hydrogen ${ }^{7}$. It should be noted that in lithium polyhydrides the metallization does not occur due to 'precompression' of hydrogen, but rather due to 'doping' by electropositive elements ${ }^{5,6}$. In extension of these ideas, recent theoretical analysis of $\mathrm{MH}_{\mathrm{n}}(\mathrm{M}=\mathrm{Li}, \mathrm{Na}, \mathrm{K}, \mathrm{Rb}, \mathrm{Cs}, \mathrm{Sr})$ compounds with variable hydrogen composition resulted in prediction of stable polyhydrides of alkali and alkaline earth metals $^{5,6,8-13}$.

The compounds with more than two hydrogen atoms per alkali atom are expected to become stable at pressures as low as $25 \mathrm{GPa}$ in the case of $\mathrm{Na}$ and above $100 \mathrm{GPa}$ in the case of Li. Hydrogen-rich polyhydride phases are also predicted at high pressures for alkaline earth metals ${ }^{10,14,15}$. Such hydrogen-rich polyhydride materials are stabilized by compression, and many of them are expected to become metallic and superconducting at lower pressures than pure hydrogen. For example, high critical superconducting temperatures $\left(T_{\mathrm{c}} \sim 235 \mathrm{~K}\right)$ are predicted for polyhydrides of $\mathrm{Ca}$ (ref. 14). Moreover, the recent discovery of superconductivity in hydrogen sulfide at a record $T_{\mathrm{c}} \sim 203 \mathrm{~K}$ at high pressure $(150 \mathrm{GPa})$ has confirmed the great potential of dense hydride materials as high-temperature superconductors ${ }^{16}$. Thus, the recently predicted polyhydride compounds may pave the route to alter the electronic structure in a way that facilitates the creation of metallic superconducting materials with record high critical superconducting temperatures ${ }^{4,17}$.

One of the salient features predicted to form in polyhydrides of heavier alkali metals is a motif of linear $\mathrm{H}_{3}^{-}$ions (such ions are predicted to form in $\mathrm{RbH}_{5}$ (ref. 18) and $\mathrm{CsH}_{3}$ (ref. 11)). $\mathrm{H}_{3}^{-}$ions are known to exist in a linear configuration, while $\mathrm{H}_{3}^{+}$ions form a triangular-shaped unit ${ }^{19,20}$. Notably, triangular $\mathrm{H}_{3}^{+}$ions were predicted to be stable in the $\mathrm{H}_{5} \mathrm{Cl}$ compound ${ }^{21}$ at high pressures up to $300 \mathrm{GPa}$. The symmetric linear $\mathrm{H}_{3}^{-}$ions ${ }^{22}$ were discussed as transition states in hydrogen exchange processes of metal complexes $^{23}$. On the experimental side, $\mathrm{H}_{3}^{-}$and $\mathrm{D}_{3}^{-}$ions were observed in discharge plasmas only recently ${ }^{24}$. It should be noted here, that ambient pressure metastable metallic hydrogen phases predicted in 1972 by Browman and Kagan ${ }^{3}$ are composed of onedimensional hydrogen chains, which are similar to the chains of $\mathrm{H}_{3}^{-}$ions predicted theoretically in $\mathrm{RbH}_{5}$ (ref. 11) and $\mathrm{CsH}_{3}$ (ref. 17). The simplest model of strong correlations in a linear chain of hydrogen atoms ${ }^{25,26}$ is also based on similar equidistant chain motifs. Despite a wealth of theoretically predicted high pressure polyhydride structures, none of the predictions has been confirmed until now, except possible Li polyhidride phases. The polyhydrides of Li were reported recently, based on the measurements of the infrared absorption spectra of $\mathrm{LiH}$ by Pepin et $a^{27}$. New absorption bands observed in their work above $130 \mathrm{GPa}$ are consistent with the calculated infrared modes in $\mathrm{LiH}_{6}$ and $\mathrm{LiH}_{2}$.
The new polyhydrides of Li have been produced by compression of $\mathrm{LiH}$ in a rhenium gasket without any pressure medium. Both compounds remain optically transparent to $215 \mathrm{GPa}$, which is at odds with calculations ${ }^{28}$. The authors did not attempt to characterize their samples by $\mathrm{x}$-ray diffraction method and Raman spectroscopy, which makes it difficult to estimate if they had significant amounts of the reacted materials in the high pressure samples.

Here we report the synthesis of Na polyhydrides at pressure of $\sim 30 \mathrm{GPa}$ in laser-heated diamond anvil cell (DAC) experiments at temperatures above $2,000 \mathrm{~K}$. We were guided by ab-initio theoretical search, which yielded a number of stable $\mathrm{NaH}_{x}$ $(x=1.5-13)$ materials (Fig. 1) more favourable than those predicted previously ${ }^{8}$. In agreement with these predictions, we identified the $\mathrm{NaH}_{3}$ solid using in situ synchrotron X-ray diffraction (XRD) measurements. Moreover, both XRD and Raman spectroscopy revealed the presence of the $\mathrm{NaH}_{7}$ phase, which has a characteristic Raman band at $3,200 \mathrm{~cm}^{-1}$, suggesting the formation of $\mathrm{H}_{3}^{-}$ions. Our results therefore provide the first verification of the existence of polyhydrides of alkali metals with heterogeneous (multicenter) chemical bonding and prospects for lower pressure metallization.

\section{Results}

X-ray diffraction experiments and Raman measurements. Several experiments were performed with $\mathrm{Li}$ and $\mathrm{Na}$ samples up to $70 \mathrm{GPa}$ at room temperature. In these runs, only the formation of $\mathrm{LiH}$ and $\mathrm{NaH}$ was detected, with no indication of polyhydride phases. The results of these experiments were similar to previously reported attempts ${ }^{29}$, however, we were able to identify $\mathrm{Li}$ and $\mathrm{Na}$ metals up to $35 \mathrm{GPa}$, and $50 \mathrm{GPa}$, respectively, without complete transformation to the monohydride form. To overcome possible kinetic barriers to the formation of polyhydrides, we performed laser-heating experiments on these samples. For Li in hydrogen we were able to perform a few experiments above $50 \mathrm{GPa}$ with laser heating up to $1,900-2,000 \mathrm{~K}$, in which only the monohydride of $\mathrm{Li}(\mathrm{LiH})$ was formed. We were not able to detect any polyhydrides of Li under these conditions. Similar measurements for $\mathrm{Na}$ in hydrogen at $32 \mathrm{GPa}$ yielded a significant enhancement of the XRD signal from $\mathrm{NaH}$. Further heating of $\mathrm{Na}$ and $\mathrm{NaH}$ in $\mathrm{H}_{2}$-saturated environment to $\sim 2,100 \mathrm{~K}$ produced a laser flash that resulted in sample changes (runaway material forming a ring centred at the flash position-see inset in Fig. 3), indicating the onset of chemical reactions. The Raman spectra collected from temperature quenched sample within the reacted area showed the formation of a new material with two additional vibron peaks $\sim 4,000 \mathrm{~cm}^{-1}$, one of them softer than the pure $\mathrm{H}_{2}$ vibron, and the other one harder (Fig. 2; low-frequency Raman spectra are shown in the Supplementary Fig. 1). However, we were unable to detect a reliable XRD signal from the very tiny sample reaction area. We repeated the laser-heating experiment with a $\mathrm{NaH}$ sample loaded in the DAC with $\mathrm{H}_{2}$ and Au fragments for measuring pressure and for better coupling to the laser during heating. This experiment produced large amounts of a new phase after laser heating at $30 \mathrm{GPa}$ (an example of the XRD pattern obtained after pressure increase to $40 \mathrm{GPa}$ is shown in Supplementary Fig. 2). As the temperature was increased above $2,100 \mathrm{~K}$ the thermal runaway resulted in a very bright flash (avalanche) saturating the detector. From the brightness of the heating spot, we estimated the temperature to be in the range of $4,000-6,000 \mathrm{~K}$. We did not attempt to repeat heating due to the risk of breaking the diamonds but saved the sample for further characterization. After heating we could clearly see the change in the sample shape, indicating the sample transport within the laser-heated reaction area of $\sim 20 \mu \mathrm{m}$ in diameter. 
a

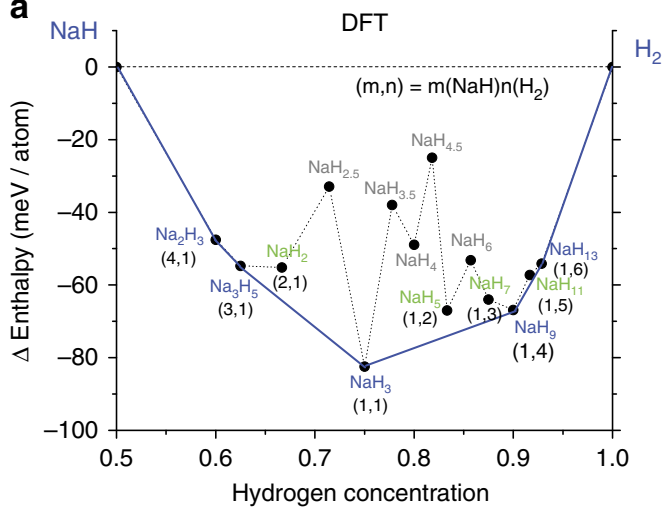

C

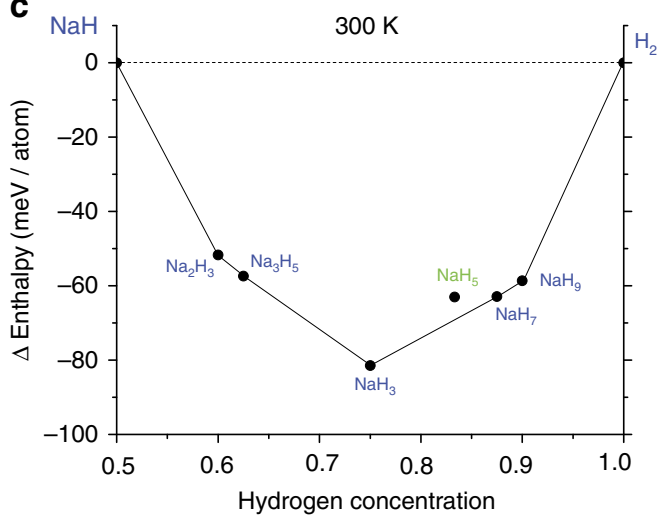

b

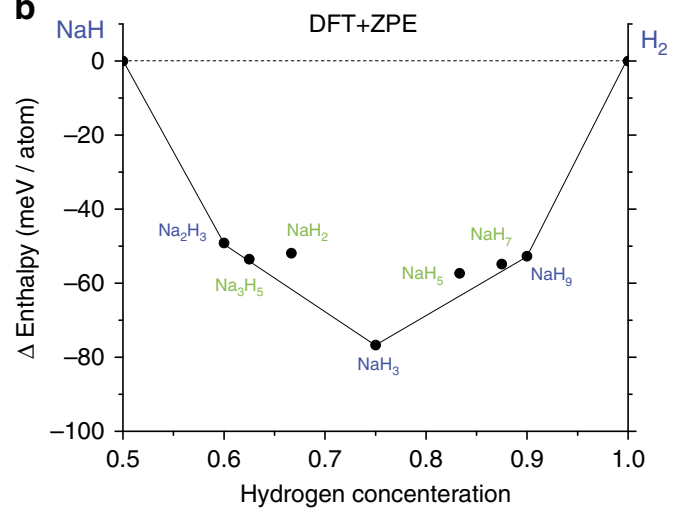

Figure 1 | Calculations of stable sodium polyhydride compounds. Convex hull curve of $\mathrm{Na}-\mathrm{H}$ compounds at $50 \mathrm{GPa}$ with respect to the decomposition (horizontal dashed line) into $\mathrm{NaH}$ and $\mathrm{H}_{2}$ using (a) density functional theory (DFT) (b) including ZPE and (c) including temperature (300 K). The chemical formula in blue (green) shows predicted stable (metastable) compounds. The $(m, n)$ correspond to compositions in units of $\mathrm{NaH}$ and $\mathrm{H}_{2}$, respectively. Chemical formulas in black are found to be stable against the decomposition into $\mathrm{NaH}$ and $\mathrm{H}_{2}$, however, they possess relatively high total energy compared to other stable (meta-stable) phases.

The newly synthesized phases were characterized by XRD and Raman measurements in the pressure range from 18 to $50 \mathrm{GPa}$. Decompression of the DAC below $18 \mathrm{GPa}$ resulted in decomposition of the newly formed phase, which was confirmed by the disappearance of their characteristic Raman signatures. These experiments are very challenging since the presence of hydrogen under high-pressure-temperature conditions often leads to diamond breakage. Most of the experiments resulted in failure of the diamond during laser heating; however, we succeeded in producing Na polyhydrides in two runs out of 10, and characterized them using Raman spectroscopy and XRD. The experimental results are described below. Before describing these results, we summarize below our theoretical findings, which differ in a number of aspects from the previous theoretical study of Baettig et al. ${ }^{8}$. These differences are crucial for understanding our experimental results.

Theoretical calculations of sodium polyhydride structures. We searched for low-enthalpy structures using a variety of compositions of $\mathrm{Na}-\mathrm{H}$ at $50 \mathrm{GPa}$ with the ab-initio Random structure searching (AIRSS) method $^{6}$, which has previously been applied to hydrides under pressure ${ }^{6,30}$. The calculations used density functional theory ${ }^{31,32}$ and the generalized gradient approximation of Perdew, Burke and Ernzerhof for the exchange-correlation functional $^{33,34}$. AIRSS was conducted at $50 \mathrm{GPa}$ with the Cambridge serial total energy package (CASTEP) plane-wave $\operatorname{code}^{35}$ and ultrasoft pseudopotentials ${ }^{36}$. Further details are provided in the Methods section.

We performed calculations for the structures reported by Baettig et al. ${ }^{8}$ and successfully reproduced their data for $\mathrm{NaH}_{7}, \mathrm{NaH}_{9}$ and
$\mathrm{NaH}_{11}$. We used AIRSS to study other compositions and we found the $\mathrm{NaH}_{3}$ phase. This prompted us to extend our searches to lower hydrogen compositions such as $\mathrm{NaH}_{2}, \mathrm{Na}_{3} \mathrm{H}_{5}$ and $\mathrm{Na}_{2} \mathrm{H}_{3}$. For most compositions, we studied simulation cells containing 1,2 and 4 formula units, and for $\mathrm{NaH}_{2}$ and $\mathrm{NaH}_{3}$, we conducted AIRSS on up to 6 formula units. The most stable materials found consisted of $\mathrm{H}_{2}$ and $\mathrm{NaH}$ structural units. This finding led us to generalize the form of the stable composition to $(\mathrm{NaH})_{m}\left(\mathrm{H}_{2}\right)_{n}$ (Fig. 1). We studied $(m, n)$ pairs ranging from $(4,1)$ to $(1,6)$. We also tested other compositions such as $\mathrm{Na}_{2} \mathrm{H}_{5}, \mathrm{Na}_{2} \mathrm{H}_{7}$ and $\mathrm{Na}_{2} \mathrm{H}_{9}$, but we found them to be unstable with respect to decomposition into nearby stable compositions, as shown in Fig. 1(a). Previous theoretical work suggested that $\mathrm{NaH}_{n}(n>6)$ can be stabilized above $50 \mathrm{GPa}$ (ref. 8). As shown in the convex hull diagram of Fig. 1 at $50 \mathrm{GPa}$, generally, many combinations of $(\mathrm{NaH})$ and $\mathrm{H}_{2}$ can be stabilized. The $\mathrm{Na}_{2} \mathrm{H}_{3}, \mathrm{Na}_{3} \mathrm{H}_{5}, \mathrm{NaH}_{3}, \mathrm{NaH}_{9}$ and $\mathrm{NaH}_{13}$ phases (shown in blue) lie on the convex hull at $50 \mathrm{GPa}$. In addition, although they are not thermodynamically stable, $\mathrm{NaH}_{2}$, $\mathrm{NaH}_{5}, \mathrm{NaH}_{7}$ and $\mathrm{NaH}_{11}$ (shown in green) are dynamically stable as demonstrated by the phonon dispersion data (corresponding structures, phonon and electron DOS are shown in Supplementary Figs 3-29 and in the paper of Baettig et al. ${ }^{8}$.

The enthalpy differences between the thermodynamically stable phases (blue line) and the dynamically stable phases (green) are only $\sim 10 \mathrm{meV}$ per atom. We also calculated the nuclear zero-point energy (ZPE) within the harmonic approximation to estimate the effects of vibrations on the total enthalpy. We found a monotonic increase in the ZPE with the fraction of $\mathrm{H}$ atoms in the various hydrides ranging from $150 \mathrm{meV}$ per atom in $\mathrm{NaH}$ to $\sim 240 \mathrm{meV}$ per atom in $\mathrm{H}_{2}$ (Supplementary Fig. 30 ). 

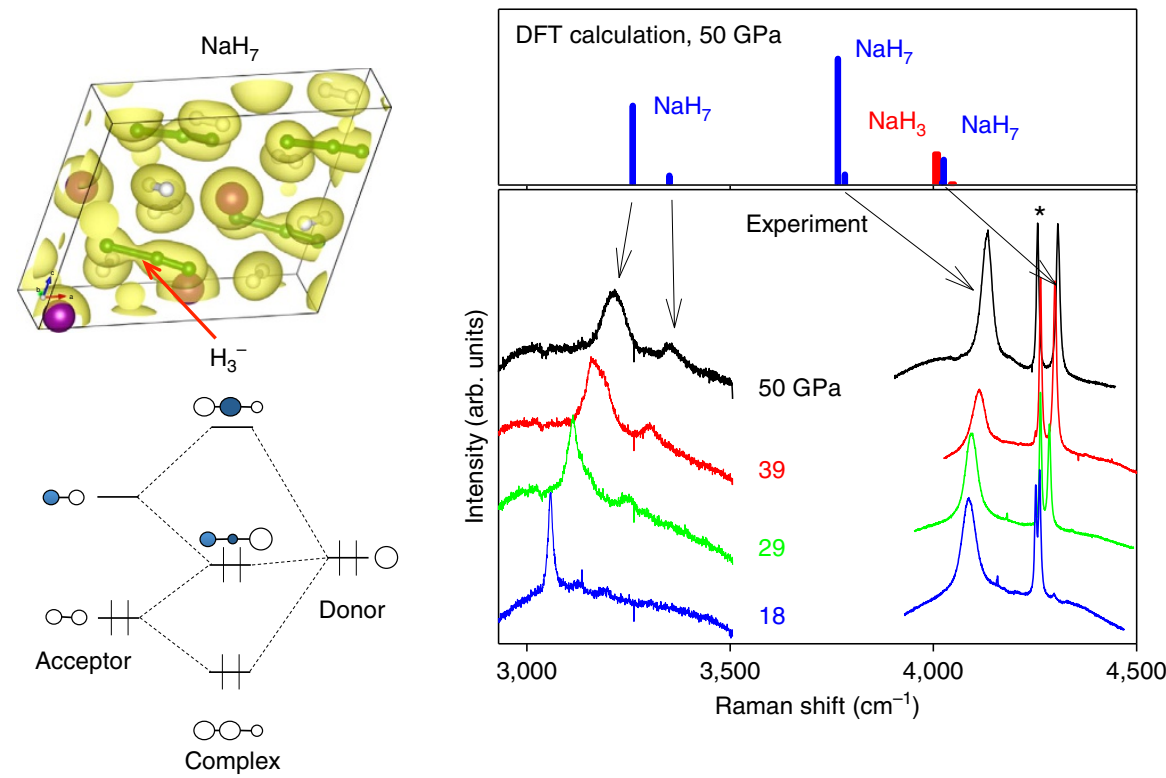

Figure 2 | $\mathbf{H}_{\mathbf{3}}^{-}$complexes in $\mathbf{N a H}_{\mathbf{7}}$ and $\mathbf{R a m a n}$ spectra of $\mathbf{N a H}_{\mathbf{3}}$ and $\mathbf{N a H}_{\mathbf{7}}$. The right panel shows higher-frequency vibrons from $\mathrm{H}_{2}$ molecular-type structural units. The left panel shows the structure of $\mathrm{NaH}_{7}$, which contains $\mathrm{H}_{3}^{-}$complexes. The isosurface is plotted at the level of 0.07 electrons $* \AA-3$. One of $\mathrm{H}_{2}$ molecules is bonded to a hydrogen atom in the $\mathrm{NaH}$ unit with a bond length of $z=1.25 \AA$, and they form a $\mathrm{H}_{3}^{-}$linear anion in $\mathrm{NaH}_{x}$ materials with $x=7$. A detailed charge analysis is presented below (Fig. 6). The schematic diagram for $\mathrm{H}_{3}^{-}$molecular orbitals (adopted from ref. 48 for $\mathrm{I}_{3}^{-}$) is also shown. Donor stands for the hydride ion $\mathrm{H}^{-}$, and acceptor for the $\mathrm{H}_{2}$ unit attached to $\mathrm{H}^{-}$. Right panel: Raman spectra of the $\mathrm{NaH}_{7}$ sample are shown in the frequency range $\left(3,000-3,500 \mathrm{~cm}^{-1}\right)$ typical for vibrons from $\mathrm{H}_{3}^{-}$units (indicated in the structure of $\mathrm{NaH}_{7}$ as green-yellow dumbells). The Raman response in 4,000-4,300 $\mathrm{cm}^{-1}$ region is a mixture of $\mathrm{H}_{2}$ vibron modes of $\mathrm{NaH}_{3}$ and $\mathrm{NaH}_{7}$. The top panel shows the calculated Raman frequencies and intensities for $\mathrm{NaH}_{3}$ and $\mathrm{NaH}_{7}$. The Raman signal from a pure $\mathrm{H}_{2}$ vibron is indicated by an asterisk.

Figure 1(a) shows that $\mathrm{NaH}_{7}$ is not thermodynamically stable but in our calculations including ZPE effect (Fig. 1(b)), we found that $\mathrm{NaH}_{7}$ comes within 1-2 meV per atom of being thermodynamically stable. The stability of $\mathrm{NaH}_{7}$ relative to other stable phases increases with temperature, and in Fig. 1(c), we show that $\mathrm{NaH}_{7}$ eventually becomes a thermodynamically stable phase at $300 \mathrm{~K}$. The temperature corrections for different polyhydride phases are summarized in Supplementary Fig. 31.

Analysis of synthesized sodium polyhydride phases. The Raman spectra of the $\mathrm{NaH}_{n}$ materials synthesized by laser heating (Fig. 2) show a number of features, which are distinct from those of the pure hydrogen within the same sample chamber under the same pressure $(50 \mathrm{GPa})$. New modes that are observed at $4,100 \mathrm{~cm}^{-1}$ and $4,200 \mathrm{~cm}^{-1}$ bracket the $\mathrm{H}_{2}$ vibron at $4,160 \mathrm{~cm}^{-1}$ and point to the formation of a new phase containing $\mathrm{H}_{2}$ molecules embedded within the sodium polyhydrite crystal structure. Moreover, as shown in Fig. 2, another set of Raman modes appears around $3,200 \mathrm{~cm}^{-}$, suggesting a strongly modified $\mathrm{H}_{2}$ species, possibly similar to the predicted $\mathrm{H}_{3}^{-}$species or molecules in polyhydrides of Cs (ref. 11) or K (ref. 9). Similar or even lower Raman frequencies are characteristic of dihydrogen moieties observed in transition metal complexes ${ }^{37,38}$ and other chemical environments ${ }^{39}$. The low-frequency region of the Raman spectra (Supplementary Fig. 1) also suggests a structure very different from pure hydrogen (for example, ref. 40) and the initial bodycentred cubic (bcc) $\mathrm{NaH}$ monohydride, which is not expected to have any allowed first order Raman active modes. Indeed, our Raman measurements for unreacted sample regions in the DAC did not produce any Raman signatures of $\mathrm{NaH}$, but indicated the presence of pure solid $\mathrm{H}_{2}$, judging from its characteristic vibron and roton bands. The low-frequency Raman spectrum of the newly synthesized material consists of strongly pressuredependent bands at $200-800 \mathrm{~cm}^{-1}$, which we identify as lattice modes in contrast to weakly pressure-dependent rotational modes of pure $\mathrm{H}_{2}$. (Supplementary Fig. 1)

Figures 3 and 4 show an XRD pattern of a new material at $40 \mathrm{GPa}$. XRD data were also obtained away from the reacted area at each pressure (see inset to Fig. 3). Three different 'families' of reflections from different phases were observed to coexist in the XRD patterns of the reacted area: (i) the unreacted bcc NaH (ambient pressure face-centred cubic (fcc) NaH transforms to bcc at $29 \mathrm{GPa}$ (ref. 41)), (ii) the fcc Au used as a pressure marker and as a laser absorber and (iii) the synthesized $\mathrm{NaH}_{n}$. To fully identify the reflections from the synthesized $\mathrm{NaH}_{n}$, we performed a detailed comparison of the XRD patterns on and away from the reacted area. A typical example is shown in Fig. 3. The positions of all reflections attributed to $\mathrm{NaH}$ and $\mathrm{Au}$ are in full agreement with the known diffraction peaks of bcc $\mathrm{NaH}$ (ref. 41) and fcc $\mathrm{Au}$, implying the absence of a chemical reaction between $\mathrm{Au}$ and $\mathrm{H}$. The reflections of bcc $\mathrm{NaH}$ and $\mathrm{Au}$ have then been subtracted when performing the final structural refinement of the $\mathrm{NaH}_{n}$ phases (Supplementary Fig. 2). This has been performed via a Rietveld refinement only for bcc $\mathrm{NaH}$ and fcc $\mathrm{Au}$ with a subsequent subtraction of the refined peaks from the raw patterns. After all reflections not belonging to the synthesized $\mathrm{NaH}_{n}$ have been successfully identified we compared the calculated XRD patterns of the predicted stable structures with the observed ones. Full indexing-refinement of the observed reflections, without the use of the predicted phases as candidates, is very difficult for a variety of reasons. First, the XRD intensity depends almost exclusively on the positions of the $\mathrm{Na}$ atoms. Second, the large number of observed peaks suggests a lowsymmetry unit cell. Finally, the texture of the two-dimensional images of the XRD data suggests a mixture of phases. Based on this analysis, we find that $\mathrm{NaH}_{3}$ is the predominant phase of the synthesized material (Fig. 4). Indeed, all the main reflections can be indexed with the orthorhombic $\mathrm{Cmcm} \mathrm{NaH}$ cell. Moreover, 


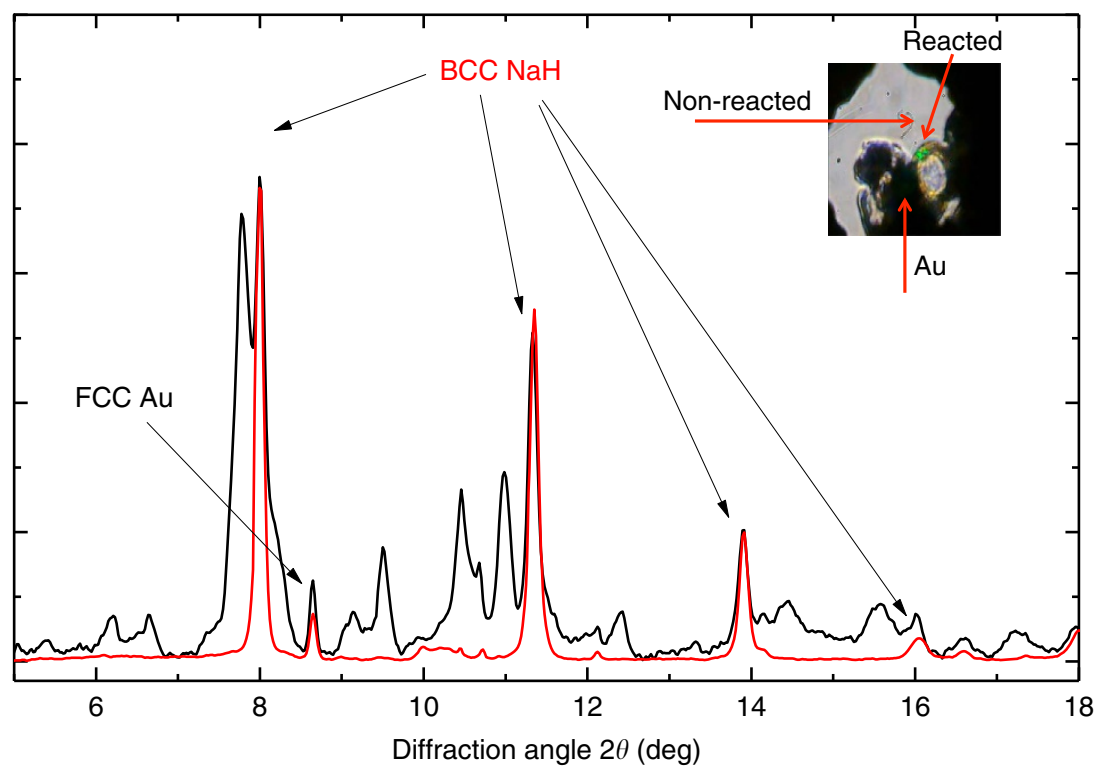

Figure 3 | Structural information from XRD measurements. XRD raw pattern (black) of the reacted area of the sample at $50 \mathrm{GPa}$, containing Bragg peaks from BCC NaH and FCC Au, which are indicated by arrows. The red XRD pattern is from a non-reacted area of the sample containing only BCC NaH and FCC Au. The perfect match of the position of the $\mathrm{NaH}$ peaks between the reacted and the non-reacted area justifies our argument about the origin of these peaks. The inset shows a reacted sample, dark sample in a gasket hole is $\mathrm{Au}+\mathrm{Na}$. The transparent part is $\mathrm{NaH}+\mathrm{H}_{2}$, the smaller dark circle with a green laser spot is a reacted area. The darker colour of the reacted area is compatible with a reduced bandgap ( $\sim 2 \mathrm{eV}$ ) obtained in the DFT calculations (Fig. 6 ).

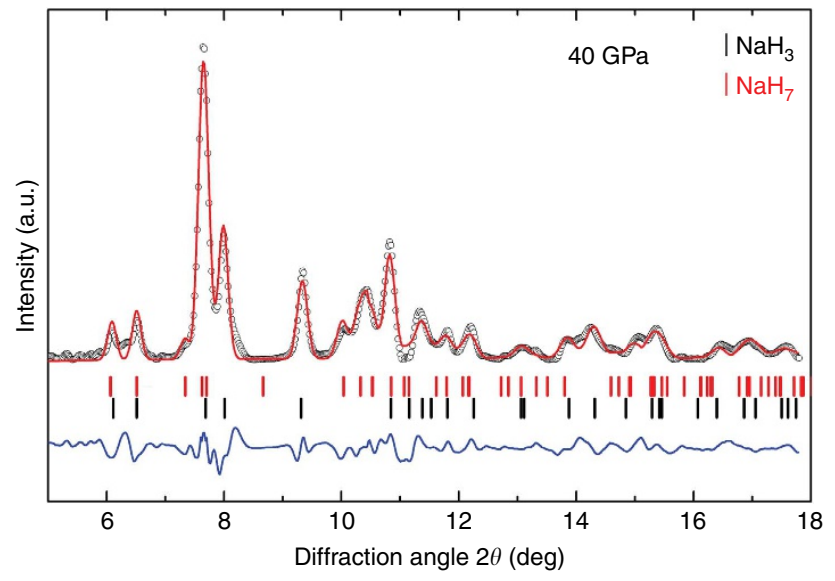

Figure 4 | Le Bail refinement for $\mathbf{N a H}_{\mathbf{n}}$ at $\mathbf{4 0} \mathbf{~ G P a}$. $\mathrm{NaH}_{3}$ and $\mathrm{NaH}_{7}$ peaks are marked with black and red vertical lines, respectively. The difference between the data and the fit is shown below (blue line).

the experimentally determined lattice parameters and cell volume (at $40 \mathrm{GPa}: \quad a=3.332 \AA, b=6.354 \AA$ and $c=4.142 \AA$ with $V_{\text {pfu }}=21.93 \AA^{3}$ ) of $\mathrm{NaH}_{3}$ are in full agreement with the theoretical predictions (Fig. 4). However, there are a few reflections that cannot be indexed with the $\mathrm{NaH}_{3}$ cell. For hydrogen contents lower than in $\mathrm{NaH}_{5}$, the phonon density of states has two well-separated bands, below $1,500 \mathrm{~cm}^{-1}$ for $\mathrm{Na}-\mathrm{H}$ interactions and around $4,000 \mathrm{~cm}^{-1}$ for $\mathrm{H}_{2}$ vibrations. At higher hydrogen concentrations, we found the formation of other intermediate frequency bands near $3,200 \mathrm{~cm}^{-1}$. Having in mind that $\mathrm{NaH}_{n}$ phases (with $n<7$ ) cannot support the existence of Raman modes at 3,200 $\mathrm{cm}^{-1}$ (Supplementary Figs 5, 6, 8, 9, 11, $12,14,15,17,18$ and 21) we have to include phases with $n>6$ (refs 7,9$)$ in our analysis. From the various phases only the monoclinic $\mathrm{Cc} \mathrm{NaH}$ phase shows reasonable agreement with the observed patterns. Indeed, some of the main observed reflections can only be indexed with the $\mathrm{NaH}_{7}$ phase with experimental lattice parameters $a=6.99 \AA, b=3.597 \AA, c=5.541 \AA$ and $\beta=69.465^{\circ} \quad$ (theoretical values $a=6.732 \AA, \quad b=3.643 \AA$, $c=5.577 \AA$ and $\beta=69.36^{\circ}$ ) at $40 \mathrm{GPa}$. With the use of both phases, $\mathrm{NaH}_{3}$ and $\mathrm{NaH}_{7}$, we have successfully indexed all observed reflections of the synthesized mixed- $\mathrm{NaH}_{n}$ material (Fig. 4). The experimental and theoretical lattice parameters and volume are summarized as a function of pressure in Fig. 5. Notably, while the experimental volumes of $\mathrm{NaH}_{3}$ and $\mathrm{NaH}+\mathrm{H}_{2}$ are very close, the volume of $\mathrm{NaH}_{7}$ is significantly lower than that of $\mathrm{NaH}+3 \mathrm{H}_{2}$. The PV term of $\mathrm{NaH}_{3}$ is practically the same (given the experimental error in both the reported EOS of $\mathrm{NaH}$ and $\mathrm{H}_{2}$ ) with that of $\mathrm{NaH}+\mathrm{H}_{2}$. There is very good agreement between observed and theoretically predicted relative intensities of Bragg reflections. However, a refinement of the positional parameters was not possible due to the 'spotty' XRD rings. Finally, Fig. 6 provides some details of the electronic structure of new phases as follows from the theoretical analysis. The electronic density of states is compatible with insulating phase for both materials, with a reduced bandgap slightly larger than the value of $2 \mathrm{eV}$ obtained from a DFT calculation. It is well known that standard DFT method underestimates the bandgaps of most semiconductors and thus it is expected that the real band gap in $\mathrm{NaH}_{3}$ and $\mathrm{NaH}_{7}$ could be larger than the calculated one. We calculated metallization pressures for $\mathrm{NaH}_{3}$ of about $250 \mathrm{GPa}$, which are similar to those predicted for higher polyhidrides in ref. 8. For $\mathrm{NaH}_{7}$ we found that electronic density contours clearly indicate formation of H3-units-Fig. 6(c,d).

\section{Discussion}

The Raman and XRD data point to the formation of $\mathrm{Na}$ polyhydrides in the predicted stability range (above $20 \mathrm{GPa}$ ). While we were unable to isolate a single well-defined polyhydride phase, the data analysis strongly supports the existence of several phases $\left(\mathrm{NaH}_{3}\right.$ and $\mathrm{NaH}_{7}$, and possibly higher polyhydrides) in the reacted sample. Most of the theoretically predicted stable $\mathrm{Na}$ 

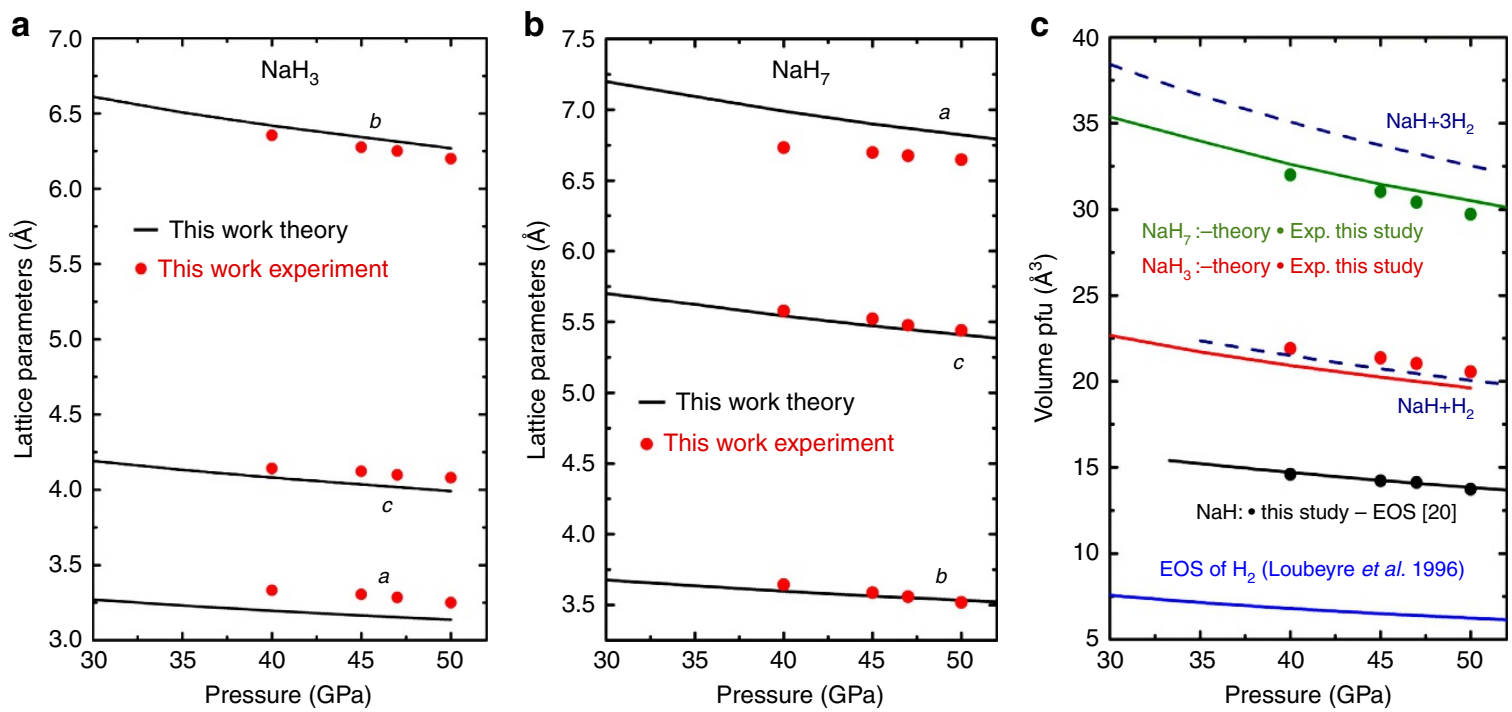

Figure 5 | Lattice parameters and equations of state of $\mathbf{N a H}_{\mathbf{3}}$ and $\mathbf{N a H}_{\mathbf{7}}$. (a) Lattice parameters of $\mathrm{NaH}_{3}$ as function of pressure. (b) Lattice parameters of $\mathrm{NaH}_{7}$ as function of pressure. (c) Equations of state (EOS) of $\mathrm{NaH}_{3}, \mathrm{NaH}_{7}$ in comparison with EOS of $\mathrm{NaH}$ and $\mathrm{H}_{2}$. Experimental data: green, red and black circles, theoretical predictions: green, red and black continuous lines (specified in the figure). EOS of $\mathrm{H}_{2}$ is also shown (blue line).
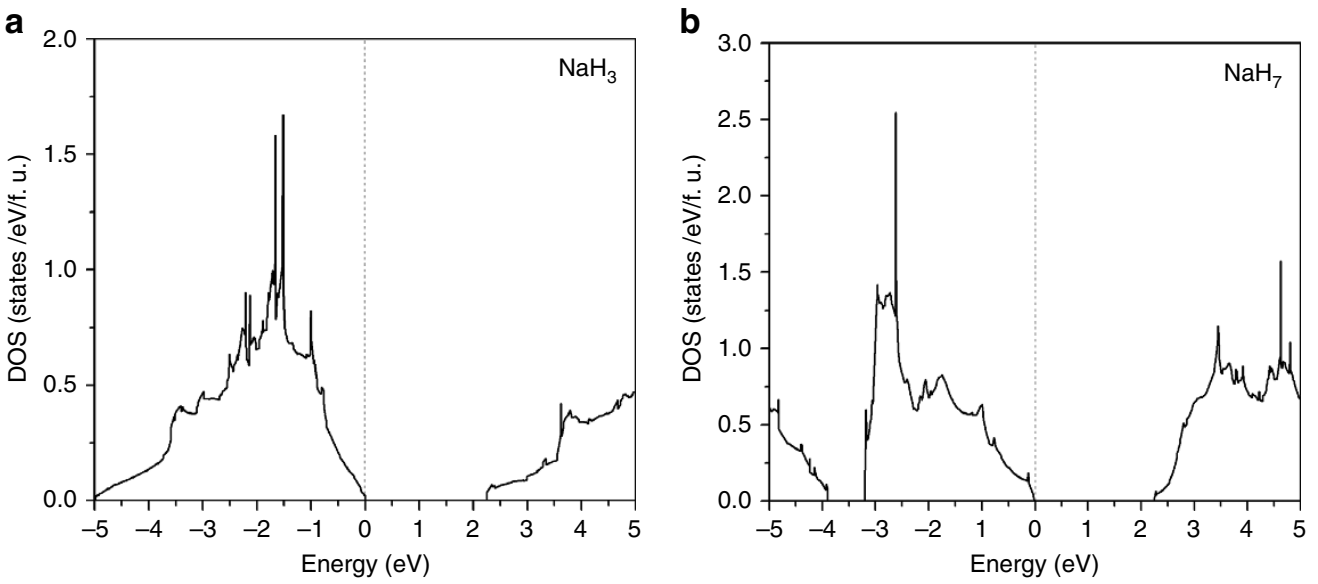

C

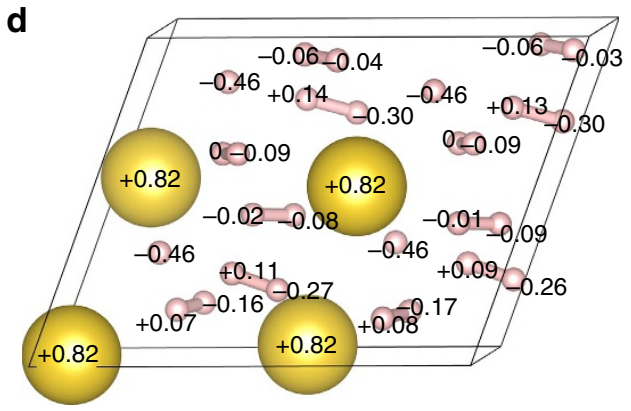

Figure 6 | Calculated electronic properties of $\mathbf{N a H}_{\mathbf{3}}$ and $\mathbf{N a H}_{\mathbf{7}}$ at $\mathbf{5 0} \mathbf{~ G P a}$. Density of electronic states of $\mathrm{NaH}_{3}(\mathbf{a})$ and $\mathrm{NaH}_{7}(\mathbf{b})$. (c) A contour plot of $\mathrm{H}^{-}$- unit in $\mathrm{NaH}_{7}$. This image shows a charge density contour with a saturation level of 0.3 electrons $\AA^{-3}$ (which is much higher than 0.07 of the isosurface plot in Fig. 2). An equi-charge density level of $\mathrm{H}^{-}$unit is evident from the plot, which was prepared for the Miller indices (1 $\left.2-1\right)$. (d) Bader analysis showing excessive charge of individual atoms in $\mathrm{NaH}_{7}$. Na cations have a charge +0.82 and ionic linked hydride $\mathrm{H}$ has a charge of -0.46 . $\mathrm{H}_{2}$ molecules with higher vibron frequencies have less polarized charges (they form pairs with charges $-0.06 \&-0.04,-0.06 \&-0.03,0 \&-0.09$, $-0.02 \&-0.08,+0.07 \&-0.16)$. However, the $\mathrm{H}_{2}$ molecules which are linked to the hydride ion $\mathrm{H}(-0.46)$ are highly polarized $(+0.14 \&-0.30$, $+0.13 \&-0.30,+0.11 \&-0.27,+0.09 \&-0.26)$.

polyhydride phases have low-symmetry structures, which are extremely difficult to characterize by XRD from the small samples available in the laser-heated region. While prolonged laser heating at well-defined $P-T$ conditions may be beneficial for growing a single-phase sample, such experiments are still inaccessible due to the high reactivity of hot hydrogen with diamond anvils. 
Notably, Raman spectroscopy provided a more sensitive tool than XRD for characterizing the formation of small amounts of low-Z polyhydride materials. Based on the results of theoretical calculations, we found that the Raman bands observed experimentally near $3,200 \mathrm{~cm}^{-1}$ can be assigned to an extended hydrogen molecular $\mathrm{H}_{2}$ unit with an intramolecular length $d$ of $\sim 0.82 \AA$. This $\mathrm{H}_{2}$ molecule is linked to a hydrogen atom in the $\mathrm{NaH}$ unit with a distance of $z=1.25 \AA$ by sharing valence electrons (Fig. 6(c,d)), and they form a $\mathrm{H}_{3}^{-}$linear anion in $\mathrm{NaH}_{x}$ materials with $x=7$ (Figs 2 and 6). It was suggested that pressure can induce a linear geometry for $\mathrm{H}_{3}^{-}$, which has four electrons, but a triangular geometry for $\mathrm{H}_{3}^{+}$, which has two electrons ${ }^{20}$; recent confirmation of these simple chemical arguments was provided by a careful theoretical study of heavy alkali-metal hydrides under pressure predicted to form linear $\mathrm{H}_{3}^{-}$in $\mathrm{KH}_{5}$. To gain further insights into $\mathrm{H}_{3}^{-}$anion formation in $\mathrm{NaH}_{7}$, we analysed the charge density of $\mathrm{NaH}_{3}$ and $\mathrm{NaH}_{7}$ using Bader analysis (Fig. 6). The calculations confirmed the highly ionic nature of the $\mathrm{NaH}$ unit in each polyhydride: the net charges on $\mathrm{Na}$ and $\mathrm{H}$ in the $\mathrm{NaH}$ unit are $+0.79 /+0.82$ and $-0.65 /-0.47$ in $\mathrm{NaH}_{3} / \mathrm{NaH}_{7}$, respectively, indicating that a significant portion of the electron density is donated to the $\mathrm{H}_{2}$ molecules in $\mathrm{NaH}_{7}$. In fact, the $\mathrm{H} 3$-anion in $\mathrm{NaH}_{7}$ has an excess of -0.63 electrons and accordingly, $\mathrm{H}_{2}$ in $\mathrm{H} 3$-anion possesses $-0.16 \mathrm{e}$, which leads to the elongation of the $\mathrm{H}_{2}$ bond.

$A b$ initio phonon calculations give information on the dynamical stability of the phases. The stability region of $\mathrm{NaH}_{7}$ was predicted ${ }^{8}$ to be $25-100 \mathrm{GPa}$ which is consistent with our experiments. All lattice and vibron modes of the polyhydrides increase monotonically in frequency with pressure up to $50 \mathrm{GPa}$. Our theoretical calculations show dynamical stability and structural stability of predicted phases, including $\mathrm{NaH}_{3}$ and $\mathrm{NaH}_{7}$.

In summary, we synthesized polyhydrides of $\mathrm{Na}$ in a laser-heated DAC at pressures above $30 \mathrm{GPa}$ and temperatures above $2,000 \mathrm{~K}$. We also performed detailed theoretical studies and found new stable phases of Na polyhydrides. One of these phases, $\mathrm{NaH}_{3}$, provides a good match to the XRD patterns collected from the reacted region. However, the $\mathrm{x}$-ray patterns also suggest the existence of higher polyhydrydes $\left(\mathrm{NaH}_{\mathrm{n}}, n \geq 7\right)$, which is supported by the analysis of the Raman spectra in the $3,200 \mathrm{~cm}^{-1}$ region. Notably, higher polyhydrides of sodium appear to stabilize the $\mathrm{H}_{3}^{-}$unit predicted for other, heavier alkali metals ${ }^{18}$. Polyhydrides of alkali metals provide a new class of materials with pressure-stabilized multicenter (3 center-4 electron) bonds for future investigation. Polyhydrides may provide chemical means to pre-compress hydrogen molecules and facilitate the creation of metallic superconducting hydrogen at reduced pressures. The possibility of metastable phases should be carefully explored in future studies, since the new polyhydrides may be implemented as hydrogen storage materials with hitherto unexplored physical and chemical properties.

\section{Methods}

High-pressure experiments. We have studied the formation of $\mathrm{Li}$ and $\mathrm{Na}$ polyhydrides in a DAC at pressures up to $70 \mathrm{GPa}$ with laser heating to $2,000 \mathrm{~K}$ and higher temperatures. The experiments were performed in a symmetric DAC (ref. 42). The samples of $\mathrm{Li}, \mathrm{Na}, \mathrm{LiH}$ and $\mathrm{NaH}$ were loaded, along with small fragments of $\mathrm{Au}$, in a glove box with controlled atmosphere (<1 p.p.m. of oxygen). According to recent experimental ${ }^{43}$ and theoretical ${ }^{44}$ results, no chemical reaction is expected between $\mathrm{Au}$ and $\mathrm{H}_{2}$, up to the highest pressure of this study. Each sample was sealed in a DAC inside a glove box, and transferred to a gas-loading apparatus, where a $\mathrm{H}_{2}$ pressure of $\sim 200 \mathrm{MPa}$ was created. The DAC was opened under the $\mathrm{H}_{2}$ pressure to let the gas in, resealed and then taken out for further high-pressure experiments.

XRD measurements and on-line laser heating were performed at the Sector 13 (GSECARS), Advanced Photon Source at the Argonne National Laboratory ${ }^{45}$. The DAC was cooled below $200 \mathrm{~K}$ with a nitrogen jet from Cryostream-type unit manufactured by Oxford Cryosystems.
}

Raman measurements were performed using off-line custom-made Raman system at GSECARS, the data were taken with Ar ion laser excitation (wavelength $514.5 \mathrm{~nm})$.

Theoretical calculations. We used the CASTEP plane-wave-basis set cutoff energy of $1,000 \mathrm{eV}$ and a Brillouin-zone integration grid of spacing $2 \pi \times 0.05 \AA^{-1}$ Phonon calculations were performed with density functional perturbation theory using the Quantum Espresso code ${ }^{46}$ with a kinetic energy cutoff of $70 \mathrm{Ry}$. The BZ integrations in the calculations were performed using Monkhorst-Pack meshes ${ }^{47}$. We refer to meshes of k-points for electronic structure calculations and meshes of q-points for phonons. The phonon calculations used $24 \times 24 \times 24$ k-points mesh and $8 \times 8 \times 8$ q-points mesh for the most studied $\mathrm{Na}-\mathrm{H}$ compounds and $12 \times 12 \times 12 \mathrm{k}$-points with a $6 \times 6 \times 6$ q-points mesh is used for relatively larger unit-cell compounds $\left(\mathrm{Na}_{2} \mathrm{H}_{3}\right.$ and $\left.\mathrm{Na}_{3} \mathrm{H}_{5}\right)$. Further details regarding the theoretical calculations are available in the Supplementary Methods.

Data availability. The authors declare that most of the data supporting the findings of this study are available within the article and its Supplementary Information Files. Any additional relevant data are available from the corresponding author on request.

\section{References}

1. Yang, J., Sudik, A., Wolverton, C. \& Siegel, D. J. High capacity hydrogen storage materials: attributes for automotive applications and techniques for materials discovery. Chem. Soc. Rev. 39, 656-675 (2010).

2. Babaev, E., Sudbø, A. \& Ashcroft, N. W. A superconductor to superfluid phase transition in liquid metallic hydrogen. Nature 431, 666-668 (2004).

3. Brovman, E. G., Kagan, Y. \& Kholas, A. Structure of metalic hydrogen at zero pressure. Sov. Phys. JETP 34, 1300-1315 (1972).

4. Ashcroft, N. W. Hydrogen dominant metallic alloys: high temperature superconductors? Phys. Rev. Lett. 92, 187002 (2004).

5. Zurek, E., Hoffmann, R., Ashcroft, N. W., Oganov, A. R. \& Lyakhov, A. O. A little bit of lithium does a lot for hydrogen. Proc. Natl Acad. Sci. USA 106, 17640-17643 (2009).

6. Pickard, C. J. \& Needs, R. J. Ab initio random structure searching. J. Phys.Condens. Matter 23, 053201 (2011).

7. McMahon, J. M. \& Ceperley, D. M. Ground-state structures of atomic metallic hydrogen. Phys. Rev. Lett. 106, 165302 (2011).

8. Baettig, P. \& Zurek, E. Pressure-stabilized sodium polyhydrides: NaH_\{n\} (n\&gt;1). Phys. Rev. Lett. 106, 237002 (2011).

9. Hooper, J. \& Zurek, E. High pressure potassium polyhydrides: a chemical perspective. J. Phys. Chem. C 116, 13322-13328 (2012)

10. Hooper, J., Altintas, B., Shamp, A. \& Zurek, E. Polyhydrides of the alkaline earth metals: a look at the extremes under pressure. J. Phys. Chem. C 117, 2982-2992 (2013).

11. Shamp, A., Hooper, J. \& Zurek, E. Compressed cesium polyhydrides: Cs ${ }^{+}$ sublattices and H3 - three-connected nets. Inorg. Chem. 51, 9333-9342 (2012).

12. Hooper, J., Terpstra, T., Shamp, A. \& Zurek, E. Composition and constitution of compressed strontium polyhydrides. J. Phys. Chem. C 118, 6433-6447 (2014).

13. Zhou, D. et al. Ab initio study revealing a layered structure in hydrogen-rich $\mathrm{KH}_{6}$ under high pressure. Phys. Rev. B 86, 014118 (2012).

14. Wang, H., Tse, J. S., Tanaka, K., Iitaka, T. \& Ma, Y. Superconductive sodalitelike clathrate calcium hydride at high pressures. Proc. Natl Acad. Sci. USA 109, 6463-6466 (2012).

15. Lonie, D. C., Hooper, J., Altintas, B. \& Zurek, E. Metallization of magnesium polyhydrides under pressure. Phys. Rev. B 87, 054107 (2013).

16. Drozdov, A. P., Eremets, M. I., Troyan, I. A., Ksenofontov, V. \& Shylin, S. I. Conventional superconductivity at 203 kelvin at high pressures in the sulfur hydride system. Nature 525, 73-76 (2015).

17. Ashcroft, N. W. Metallic hydrogen: a high-temperature superconductor? Phys Rev. Lett. 21, 1748-1749 (1968).

18. Hooper, J. \& Zurek, E. Rubidium polyhydrides under pressure: emergence of the linear $\mathrm{H}_{3}$ - species. Chemistry 18, 5013-5021 (2012).

19. Coulson, C. A. The electronic structure of $\mathrm{H}_{3}+$. Proc. Camb. Philos. Soc. 31, 244-259 (1935).

20. Grochala, W., Hoffmann, R., Feng, J. \& Ashcroft, N. W. The chemical imagination at work in very tight places. Angew. Chem. Int. Ed. 46, 3620-3642 (2007).

21. Wang, Z., Wang, H., Tse, J. S., Iitaka, T. \& Ma, Y. Stabilization of $\mathrm{H}_{3}+$ in the high pressure crystalline structure of $\mathrm{HnCl}(\mathrm{n}=2-7)$. Chem. Sci. 6, 522-526 (2015).

22. Ayouz, M., Dulieu, O., Guérout, R., Robert, J. \& Kokoouline, V. Potential energy and dipole moment surfaces of $\mathrm{H}_{3}-$ molecule. J. Chem. Phys. 132, 194309 (2010).

23. Maseras, F., Lledós, A., Clot, E. \& Eisenstein, O. Transition metal polyhydrides: from qualitative ideas to reliable computational studies. Chem. Rev. 100, 601-636 (2000). 
24. Wang, W. et al. Observations of $\mathrm{H}_{3}-$ and $\mathrm{D}_{3}-$ from dielectric barrier discharge plasmas. Chem. Phys. Lett. 377, 512-518 (2003).

25. Mazziotti, D. A. Large-Scale semidefinite programming for many-electron quantum mechanics. Phys. Rev. Lett. 106, 083001 (2011).

26. Stella, L., Attaccalite, C., Sorella, S. \& Rubio, A. Strong electronic correlation in the hydrogen chain: A variational Monte Carlo study. Phys. Rev. B 84, 245117 (2011).

27. Pépin, C., Loubeyre, P., Occelli, F. \& Dumas, P. Synthesis of lithium polyhydrides above $130 \mathrm{GPa}$ at 300K. Proc. Natl Acad. Sci. USA 112, 7673-7676 (2015).

28. Xie, Y., Li, Q., Oganov, A. R. \& Wang, H. Superconductivity of lithium-doped hydrogen under high pressure. Acta Crystallogr. Sect. C 70, 104-111 (2014).

29. Howie, R. T., Narygina, O., Guillaume, C. L., Evans, S. \& Gregoryanz, E. Highpressure synthesis of lithium hydride. Phys. Rev. B 86, 064108 (2012).

30. Pickard, C. J. \& Needs, R. J. High-pressure phases of silane. Phys. Rev. Lett. 97, 045504 (2006)

31. Hohenberg, P. \& Kohn, W. Inhomogeneous electron gas. Phys. Rev. 136, B864-B871 (1964)

32. Kohn, W. \& Sham, L. J. Self-consistent equations including exchange and correlation effects. Phys. Rev. 140, A1133-A1138 (1965).

33. Perdew, J. P. et al. Atoms, molecules, solids, and surfaces: applications of the generalized gradient approximation for exchange and correlation. Phys. Rev. B 46, 6671-6687 (1992).

34. Perdew, J. P., Burke, K. \& Ernzerhof, M. Generalized gradient approximation made simple. Phys. Rev. Lett. 77, 3865-3868 (1996).

35. Clark, S. J. et al. First principles methods using CASTEP. Z. Kristallogr. 220, 567-570 (2005).

36. Vanderbilt, D. Soft self-consistent pseudopotentials in a generalized eigenvalue formalism. Phys. Rev. B 41, $7892-7895$ (1990).

37. Kubas, G. J., Ryan, R. R., Swanson, B. I., Vergamini, P. J. \& Wasserman, H. J. Characterization of the first examples of isolable molecular hydrogen complexes, $\mathrm{M}(\mathrm{CO})_{3}\left(\mathrm{PR}_{3}\right)_{2}\left(\mathrm{H}_{2}\right)(\mathrm{M}=$ molybdenum or tungsten; $\mathrm{R}=\mathrm{Cy}$ or isopropyl). Evidence for a side-on bonded dihydrogen ligand. J. Am. Chem. Soc. 106, 451-452 (1984).

38. Kubas, G. J. Dihydrogen complexes as prototypes for the coordination chemistry of saturated molecules. Proc. Natl Acad. Sci. USA 104, 6901-6907 (2007).

39. Richardson, T., de Gala, S., Crabtree, R. H. \& Siegbahn, P. E. M. Unconventional hydrogen bonds: intermolecular B-H $\cdots \mathrm{H}-\mathrm{N}$ interactions. J. Am. Chem. Soc. 117, 12875-12876 (1995).

40. Mao, H.-K. \& Hemley, R. J. Ultrahigh-pressure transitions in solid hydrogen. Rev. Mod. Phys. 66, 671-692 (1994).

41. Duclos, S. J., Vohra, Y. K., Ruoff, A. L., Filipek, S. \& Baranowski, B. High-pressure studies of $\mathrm{NaH}$ to $54 \mathrm{GPa}$. Phys. Rev. B 36, 7664-7667 (1987).

42. Mao, H. K., Shen, G., Hemley, R. J. \& Duffy, T. S. in Properties of Earth and Planetary Materials at High Pressure and Temperature Vol. 101 (eds Manghnani, M. H. \& Yagi, T.) 27 (Washington, DC, USA, 1998).

43. Donnerer, C., Scheler, T. \& Gregoryanz, E. High-pressure synthesis of noble metal hydrides. J. Chem. Phys. 138, 134507 (2013).

44. Kim, D. Y., Scheicher, R. H., Pickard, C. J., Needs, R. J. \& Ahuja, R. Predicted formation of superconducting platinum-hydride crystals under pressure in the presence of molecular hydrogen. Phys. Rev. Lett. 107, 117002 (2011).

45. Prakapenka, V. B. et al. Advanced flat top laser heating system for high pressure research at GSECARS: application to the melting behavior of germanium. High Press. Res. 28, 225-235 (2008).

46. Baroni, S., de Gironcoli, S., Dal Corso, A. \& Giannozzi, P. Phonons and related crystal properties from density-functional perturbation theory. Rev. Mod. Phys. 73, 515-562 (2001).

47. Monkhorst, H. J. \& Pack, J. D. Special points for Brillouin-zone integrations. Phys. Rev. B 13, 5188-5192 (1976).

48. Landrum, G. A., Goldberg, N. \& Hoffmann, R. Bonding in the trihalides $\left(\mathrm{X}_{3}-\right)$, mixed trihalides $\left(\mathrm{X}_{2} \mathrm{Y}-\right)$ and hydrogen bihalides $\left(\mathrm{X}_{3} \mathrm{H}-\right)$. The connection between hypervalent, electron-rich three-center, donor-acceptor and strong hydrogen bonding [double dagger]. J. Chem. Soc. Dalton Trans. 3605-3613 (1997).

\section{Acknowledgements}

High-pressure experiments were supported by DOE/BES under contract no. DE-FG02-02ER45955 and DE-FG02-99ER45775. D.Y.K. and T.M. acknowledge salary support by Energy Frontier Research in Extreme Environments Center (EFree), an Energy Frontier Research Center funded by the U.S. Department of Energy, Office of Science under Award Number DE-SC0001057. C.J.P. and R.J.N. were supported by the Engineering and Physical Sciences Research Council (EPSRC) of the UK. E.S. and A.F.G. acknowledge support of DARPA under contracts nos'. W31P4Q1310005 and W31P4Q1210008. R. J. N. acknowledges financial support from the Engineering and Physical Sciences Research Council (EPSRC) of the U.K. [EP/J017639/1]. C. J. P. acknowledges financial support from EPSRC [EP/G007489/2]. E.S. has performed parts of the work under the auspices of the U. S. Department of Energy by Lawrence Livermore National Security, LLC under Contract DE- AC52-07NA27344. A.F.G. acknowledges support of NSFC (no. 21473211). Portions of this work were performed at GeoSoiIEnviroCARS (Sector 13), Advanced Photon Source (APS) and Argonne National Laboratory. GeoSoilEnviroCARS is supported by the National Science FoundationEarth Sciences (EAR-1128799) and Department of Energy-Geosciences (DE-FG0294ER14466). Use of the Advanced Photon Source was supported by the U.S. Department of Energy, Office of Science and Office of Basic Energy Sciences under contract no. DEAC02-06CH11357. We thank K. Zhuravlev and S. Tkachev for help with XRD and Raman measurements at GSECARS.

\section{Author contributions}

The first two authors made equally important contributions in experiment (V.V.S.) and theoretical analysis (D.Y.K.) to the presented work. V.V.S. has developed experiment conception and design, loaded the samples in DAC, performed acquisition of data, analysis and interpretation of data, wrote the article draft and revised critically for important intellectual content at all stages; D.Y.K. performed theoretical analysis, participated in writing the article draft and revised critically for important intellectual content; E.S. participated in data acquisition, analysed XRD data and revised the paper critically for important intellectual content; T.M. loaded the samples in DAC, performed acquisition of data and revised the paper critically for important intellectual content; H.-K.M. revised article draft critically for important intellectual content at all stages; C.J.P. performed theoretical analysis, revised a paper draft critically for important intellectual content; R.J.N. performed theoretical analysis and revised a paper draft critically for important intellectual content; V.B.P. developed experiment conception and design, helped in performing acquisition of data, analysis and interpretation of data, revised a paper draft critically for important intellectual content; A.F.G. participated in acquisition of data, analysis and interpretation of data, edited article draft and revised critically for important intellectual content at all stages.

\section{Additional information}

Supplementary Information accompanies this paper at http://www.nature.com/ naturecommunications

Competing financial interests: The authors declare no competing financial interests.

Reprints and permission information is available online at http://npg.nature.com/ reprintsandpermissions/

How to cite this article: Struzhkin, V. V. et al. Synthesis of sodium polyhydrides at high pressures. Nat. Commun. 7:12267 doi: 10.1038/ncomms12267 (2016).

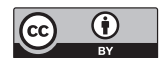

This work is licensed under a Creative Commons Attribution 4.0 International License. The images or other third party material in this article are included in the article's Creative Commons license, unless indicated otherwise in the credit line; if the material is not included under the Creative Commons license, users will need to obtain permission from the license holder to reproduce the material. To view a copy of this license, visit http://creativecommons.org/licenses/by/4.0/

(C) The Author(s) 2016 